\title{
ON CARTAN SUBALGEBRAS OF ALTERNATIVE ALGEBRAS $\left({ }^{1}\right)$
}

\author{
BY \\ D. M. FOSTER( $\left.{ }^{2}\right)$
}

\begin{abstract}
In 1966, Jacobson introduced the notion of a Cartan subalgebra for finite-dimensional Jordan algebras with unity over fields of characteristic not 2. Since finite-dimensional Jordan, alternative, and Lie algebras are known to be related through their structure theories, it would seem logical that such an analogue would also exist for finite-dimensional alternative algebras. In this paper, we show that this is the case. Moreover, the linear transformation we define that plays the role in alternative algebras that "ad ( )" plays in Lie algebras is identical with that used in the Jordan theory, and can be used in the Lie case as well. Hence we define Cartan subalgebras relative to this linear transformation for finite-dimensional alternative, Jordan, and Lie algebras, and observe that in the Lie case, they coincide with the classical definition of a Cartan subalgebra.
\end{abstract}

1. Introduction. In 1966, Jacobson [4] developed an analogue of Cartan subalgebras for finite-dimensional Jordan algebras with unity over fields of characteristic not 2. Since finite-dimensional Jordan, alternative, and Lie algebras are known to be related through their structure theories, it would seem logical that such an analogue would also exist for finite-dimensional alternative algebras independent of the fact that the symmetric algebra of an alternative algebra is a Jordan algebra.

In this paper, we show that this is indeed the case. Moreover, the linear transformation we define that plays the role in alternative algebras that "ad ( )" plays in the Lie case is identical with that used in the Jordan theory, and can be used for Lie algebras as well. Hence we define Cartan subalgebras relative to this linear transformation for finite-dimensional alternative, Jordan, and Lie algebras, and observe that in the Lie case they coincide with the classical definition of a Cartan subalgebra. However, in the Lie case as in the Jordan case, we are restricted to

Received by the editors September 21, 1970.

AMS 1970 subject classifications. Primary 17D05; Secondary 17B99, 17C99.

Key words and phrases. Universal enveloping algebra, $a$-nilpotent, $a$-nil, Engel's theorem, minimal Engel, Cartan subalgebra, $a$-regular element, inner automorphism, conjugate.

${ }^{(1)}$ Some of the results of this paper are contained in the author's doctoral dissertation written at the University of British Columbia under the supervision of Dr. C. T. Anderson.

${ }^{(2)}$ The author gratefully acknowledges the financial support of the University of British Columbia, and the hospitality of the School of Mathematics, University of Bristol, England, and the Istituto Matematico "Leonida Tonelli", Università di Pisa, Italy. The author wishes to express his gratitude to the referee for his very helpful suggestions.

Copyright (c) 1972, American Mathematical Society 
ground fields of characteristic not 2. Finally, following Barnes [1], we will see that we can characterize our Cartan subalgebras as "minimal Engel subalgebras".

Most of the notation in this paper is standard. If $U$ is an algebra and $I$ is an ideal of $U$, we write $I \triangleleft U$. If $u \in U$, by $R_{u}$ and $L_{u}$ we mean the linear transformations of $U$ where $x \in U, x R_{u}=x u$ and $x L_{u}=u x$. We recall that if $U$ is a Lie algebra, then $R_{u}=\operatorname{ad}(u)$. If $u_{1}, u_{2}, u_{3} \in U$, we write $\left(u_{1}, u_{2}, u_{3}\right)=u_{1} u_{2} \cdot u_{3}-u_{1} \cdot u_{2} u_{3}$ for the associator of $u_{1}, u_{2}$, and $u_{3}$, and $\left[u_{1}, u_{2}\right]=u_{1} u_{2}-u_{2} u_{1}$ for the commutator of $u_{1}$ and $u_{2}$.

2. Preliminaries. An algebra $A$ over a field $F$ is alternative if $(a, a, b)=(b, a, a)$ $=0$ for all elements $a, b \in A$. From this definition, it is clear that a homomorphic image of an alternative algebra and a direct sum of alternative algebras are alternative. Since the associator is multilinear, we have that $A_{K}$ is alternative for all extensions $K$ of $F$, where $A_{K}=A \otimes_{F} K$ is the scalar extension of $A$ to an algebra over $K$.

We will now give those properties of alternative algebras that we will require in our construction of Cartan subalgebras, and refer the reader to Schafer [8] for proofs.

Linearizing the identities that define $A$, we obtain

$$
\begin{aligned}
& (a, b, c)=-(a, c, b)=(c, a, b), \quad \text { for all } a, b, c \in A . \\
& (a, b, a)=0
\end{aligned}
$$

In terms of $R_{a}$ and $L_{a}, a \in A$, these become

$$
\begin{aligned}
R_{a} R_{b}-R_{a b} & =L_{a b}-L_{b} L_{a}=\left[L_{b}, R_{a}\right] \\
& =L_{a} L_{b}-L_{b a}=R_{b a}-R_{b} R_{a}=\left[R_{b}, L_{a}\right], \text { for all } a, b \in A . \\
{\left[R_{a}, L_{a}\right] } & =0
\end{aligned}
$$

Since the subalgebra of $A$ generated by any two elements of $A$ is associative, we have

$$
\begin{aligned}
& R_{a^{i}}=\left(R_{a}\right)^{i}, \\
& L_{a^{i}}=\left(L_{a}\right)^{i}
\end{aligned} \text { for all } a \in A \text { and positive integers } i .
$$

Now suppose $e$ is an idempotent of $A$. Then $A$ can be written as a direct sum

$$
\begin{aligned}
A & =A_{00} \oplus A_{10} \oplus A_{01} \oplus A_{11}, \\
A_{i j} & =\left\{x_{i j} \in A \mid x_{i j} e=j x_{i j}, e x_{i j}=i x_{i j}\right\}, \quad i, j=0,1 .
\end{aligned}
$$

If $\left\{e_{1}, \ldots, e_{t}\right\}$ is a set of pairwise orthogonal idempotents of $A\left(e_{i}^{2}=e_{i}, e_{i} e_{j}=e_{j} e_{i}=0\right.$ for $i \neq j$ ), we get a refined decomposition of $A$ as

$$
A=\sum_{i, j=1}^{t} \oplus A_{i j}
$$

where if $\delta_{i j}$ is the Kronecker delta,

$$
A_{i j}=\left\{x_{i j} \in A \mid e_{k} x_{i j}=\delta_{k i} x_{i j}, x_{i j} e_{k}=\delta_{j k} x_{i j}, k=1, \ldots, t\right\} .
$$


This decomposition is called the Pierce decomposition of $A$ relative to the set $\left\{e_{1}, \ldots, e_{t}\right\}$. We have the following multiplicative relationships between the subspaces $A_{i j}$ :

$$
\begin{array}{rlrl}
A_{i j} A_{j k} \subseteq A_{i k}, & & i, j, k=1, \ldots, t \\
A_{i j} A_{i j} \subseteq A_{j i}, & & i, j=1, \ldots, t \\
A_{i j} A_{p q}=0, & j \neq p, \quad(i, j) \neq(p, q), \quad i, j, p, q=1, \ldots, t .
\end{array}
$$

If $A$ is finite dimensional, then the following are equivalent:

(i) $A$ is nilpotent,

(ii) $A$ is solvable,

(iii) $A$ is nil.

In this case, $A$ has a unique maximal solvable ideal $S^{\prime}(A)$ and $S^{\prime}\left(A / S^{\prime}(A)\right)=0$. $S^{\prime}(A)$ is called the radical of $A$. If $S^{\prime}(A)=0, A$ is semisimple.

Finally, an idempotent $e$ of $A$ is primitive if $e$ cannot be written as a sum of nonzero pairwise orthogonal idempotents.

3. The universal enveloping algebra of an alternative algebra. Suppose $A$ is an alternative algebra over a field $F$. We will denote its anti-isomorphic image by $A^{\prime}$, and set $B=A \oplus A^{\prime}$. If $a \in A$, we will write $a^{\prime}$ for the anti-isomorphic image of $a$.

(3.1) Suppose $\rho$ is a linear map from $B$ into an associative algebra $V$ such that

$$
\begin{aligned}
& {\left[a_{1}^{\prime} \rho, a_{2} \rho\right]=\left(a_{1} a_{2}\right) \rho-\left(a_{1} \rho\right)\left(a_{2} \rho\right)} \\
& =\left(a_{2} a_{1}\right)^{\prime} \rho-\left(a_{1}^{\prime} \rho\right)\left(a_{2}^{\prime} \rho\right)=\left[a_{1} \rho, a_{2}^{\prime} \rho\right], \\
& \left(a_{1} a_{1}\right) \rho-\left(a_{1} \rho\right)\left(a_{1} \rho\right)=\left(a_{1} a_{1}\right)^{\prime} \rho-\left(a_{1}^{\prime} \rho\right)\left(a_{1}^{\prime} \rho\right)=0
\end{aligned}
$$

for all $a_{1}, a_{2} \in A$. Then $\rho$ is called a representation of $A$. We note that if the characteristic of $F$ is different from 2 , then the conditions given in (ii) are a consequence of those given in (i).

Every alternative algebra $A$ has a representation. Indeed, the linear map $\rho_{R}$ where, if $a \in A, a \rho_{R}=R_{a}$ and $a^{\prime} \rho_{R}=L_{a}$ is a representation of $A$, and is called the regular representation of $A$. Here $V$ is the ring of endomorphisms of $A$.

(3.2) Suppose $U$ is an associative algebra for which there is a linear map $i: B \rightarrow U$ such that for each representation $\rho$ of $A$ into an associative algebra $V$, there is a unique homomorphism $\rho^{*}: U \rightarrow V$ such that $b \rho=(b i) \rho^{*}$ for all $b \in B$. Then the pair $(U, i)$ is called a universal enveloping algebra for $A$.

To see that such an algebra exists, let $T(B)$ be the tensor algebra based on the vector space $B$, i.e. $T(B)=B_{1} \oplus B_{2} \oplus \cdots$ where $B_{1}=B$ and inductively for $n>1$, $B_{n}=B_{n-1} \otimes B_{1}$. The vector space operations in $T(B)$ are the usual ones, and $T(B)$ has an associative product $\otimes$ consistent with this notation. Now any representation 
$\rho$ of $A$ into an associative algebra $V$ can be uniquely extended to a homomorphism $\rho^{\prime}$ of $T(B)$ into $V$. From (3.1), the kernel of $\rho^{\prime}$ contains all elements of the form

(i) $a_{1}^{\prime} \otimes a_{2}-a_{2} \otimes a_{1}^{\prime}-a_{1} a_{2}+a_{1} \otimes a_{2}$,

(ii) $a_{1} a_{2}-a_{1} \otimes a_{2}-\left(a_{2} a_{1}\right)^{\prime}+a_{1}^{\prime} \otimes a_{2}^{\prime}$,

(iii) $\left(a_{2} a_{1}\right)^{\prime}-a_{1}^{\prime} \otimes a_{2}^{\prime}-a_{1} \otimes a_{2}^{\prime}+a_{2}^{\prime} \otimes a_{1}$,

(iv) $a_{1} a_{1}-a_{1} \otimes a_{1}$,

(v) $\left(a_{1} a_{1}\right)^{\prime}-a_{1}^{\prime} \otimes a_{1}^{\prime} \quad$ where $a_{1}, a_{2} \in A$.

Suppose $K$ is the ideal of $T(B)$ generated by elements of this form. Then $\rho^{\prime}$ will induce a unique homomorphism $\rho^{*}: T(B) / K \rightarrow V$. Hence if $i$ is the restriction to $B$ of the natural homomorphism from $T(B)$ onto $T(B) / K$, we have $b \rho=(b i) \rho^{*}$ for all $b \in B$, and it follows that $(T(B) / K, i)$ is a universal enveloping algebra of $A$. We will write $U(A)=T(B) / K$, and for each $a \in A, a i=\bar{a}$ and $a^{\prime} i=\bar{a}^{\prime}$.

(3.4) We have the following properties for the universal enveloping algebra [5, Chapter 5]:

(i) universal enveloping algebras are determined within isomorphism by $A$, consequently we take $(U(A), i)$ for the universal enveloping algebra of $A$,

(ii) $U(A)$ is generated by $\bar{a}$ and $\bar{a}^{\prime}, a \in A$,

(iii) if $K \triangleleft A$ and $D$ is the ideal of $U(A)$ generated by $\left(K \oplus K^{\prime}\right) i$, then there exists an isomorphism of $U(A / K)$ onto $U(A) / D$ such that

$$
\overline{a+\left(K \oplus K^{\prime}\right) i} \rightarrow \bar{a}+D
$$

and

$$
\overline{a^{\prime}+\left(K \oplus K^{\prime}\right) i} \rightarrow \bar{a}^{\prime}+D .
$$

We now wish to prove that if $A$ is finite dimensional and $I$ is a nil ideal of $A$, then $\left(I \oplus I^{\prime}\right) i$ generates a nilpotent ideal in $U(A)$. We begin by proving

Lemma 3.5. Suppose $I \triangleleft A$ and $I^{*}$ is the subalgebra of $U(A)$ generated by $\left(I \oplus I^{\prime}\right) i$. Then $D=I^{*}+I^{*} \otimes U(A)$ is a two-sided ideal of $U(A)$.

Proof. It is clear that $D$ is a right ideal of $U(A)$. To see that it is a left ideal, we observe from (3.3)(i)-(iii) that we have the following identities in $U(A)$ :

$$
\begin{aligned}
\text { (i) } \bar{a} \otimes \bar{b}^{\prime} & =\bar{b}^{\prime} \otimes \bar{a}-\overline{b a}+\bar{b} \otimes \bar{a}, \\
\text { (ii) } \bar{a}^{\prime} \otimes \bar{b}^{\prime} & =(\overline{b a})^{\prime}+\overline{b a}-\bar{b} \otimes \bar{a}, \\
\text { (iii) } \bar{a} \otimes \bar{b} & =\overline{a b}+\overline{b a}-\bar{b} \otimes \bar{a}, \\
\text { (iv) } \bar{a}^{\prime} \otimes \bar{b} & =\bar{b} \otimes \bar{a}^{\prime}+\bar{b} \otimes \bar{a}-\overline{b a} .
\end{aligned}
$$

Since $\bar{a}$ and $\bar{a}^{\prime}, a \in A$, generate $U(A)$, and $\bar{b}$ and $\bar{b}^{\prime}, b \in I$, generate $I^{*}$, it follows from these identities that $D$ is a two-sided ideal of $U(A)$. 
Corollary 3.7. In the setting of the previous lemma, if $I^{*}$ is nilpotent, then $D$ is nilpotent.

Proof. Since $U(A) \otimes I \subseteq D$, an easy induction argument shows that for all $n$, $D^{n} \subseteq\left(I^{*}\right)^{n}+\left(I^{*}\right)^{n} \otimes U(A)$, and the result follows.

Lemma 3.8. If $A$ is nil and the dimension of $A$ is one, then $(U(A))^{3}=0$.

Proof. Since the dimension of $A$ is one, $A=F \cdot e$ where $e^{2}=0$. By (3.3)(iv) and (v) we have $\bar{e} \otimes \bar{e}=\bar{e}^{\prime} \otimes \bar{e}^{\prime}=0$. Hence by (3.6)(i), $\bar{e} \otimes \bar{e}^{\prime}=\bar{e}^{\prime} \otimes \bar{e}$. Since $U(A)$ is generated by $\left\{\bar{e}, \bar{e}^{\prime}\right\}$, it follows easily that $(U(A))^{3}=0$.

Lemma 3.9. Suppose $A$ is finite dimensional. If $A$ is nil, then $U(A)$ is nilpotent.

Proof. The proof is by induction on the dimension of $A$. By Lemma 3.8, we may assume $\operatorname{dim} A=n>1$. Since $A$ is solvable, there is an $n-1$ dimensional subspace $I$ such that $A^{2} \subseteq I \subseteq A$, whence $I \triangleleft A$. Consequently, as $\operatorname{dim}(A / I)=1, U(A / I)$ is nilpotent. Now let $I_{1}$ be the ideal of $U(A)$ and $I^{*}$ the subalgebra of $U(A)$ generated by $\left(I \oplus I^{\prime}\right) i$. Then $I_{1}=I^{*}+I^{*} \otimes U(A)$. By the induction hypothesis, $U(I)$ is nilpotent, hence $I^{*}$, being a homomorphic image of $U(I)$, is nilpotent. By Corollary 3.7, $I_{1}$ is nilpotent. But by (3.4)(iii), $U(A / I)$ is isomorphic to $U(A) / I_{1}$, whence $U(A) / I_{1}$ is nilpotent. Therefore $U(A)$ is nilpotent, as required.

THEOREM 3.10. Suppose $A$ is finite dimensional, $(U(A), i)$ is the universal enveloping algebra of $A$, and $I$ is a nil ideal of $A$. Then $\left(I \oplus I^{\prime}\right) i$ generates a nilpotent ideal in $U(A)$.

Proof. Suppose $I^{*}$ is the subalgebra of $U(A)$ generated by $\left(I \oplus I^{\prime}\right) i$. By Lemma 3.9, $U(I)$ is nilpotent, thus $I^{*}$ is nilpotent, and the result follows from Corollary 3.7.

REMARK. Schafer has obtained what is essentially this result for finite-dimensional alternative algebras over fields of characteristic zero [7].

As a corollary to Theorem 3.10 , we prove the following result which we will require later in the paper.

Corollary 3.11. Suppose $A$ is finite dimensional, $A_{1}$ is a subalgebra of $A$, and $I$ is a nil ideal of $A_{1}$. If $C$ is the subalgebra of $\operatorname{Hom}_{F}(A, A)$ generated by $R_{a}, L_{a}, a \in A_{1}$, then $R_{b}, L_{b}, b \in I$, generate a nilpotent ideal in $C$.

Proof. Let $\rho$ be a linear map from $A_{1} \oplus A_{1}^{\prime}$ into $C$ where, if $a \in A_{1}, a \rho=R_{a}$ and $a^{\prime} \rho=L_{a}$. Then $\rho$ is a representation of $A_{1}$, hence there is a homomorphism from $U\left(A_{1}\right)$ into $C$. Since $C$ is generated by $R_{a}$ and $L_{a}, a \in A_{1}$, it follows that this homomorphism is onto $C$ and maps the ideal of $U\left(A_{1}\right)$ generated by $\left(I \oplus I^{\prime}\right) i$ onto the ideal of $C$ generated by $R_{b}, L_{b}, b \in I$. Since $\left(I \oplus I^{\prime}\right) i$ generates a nilpotent ideal in $U\left(A_{1}\right), R_{b}$ and $L_{b}, b \in I$, generate a nilpotent ideal in $C$, as desired. 
4. The theory of $a$-nilpotence.

(4.1) Suppose $A$ is an alternative algebra over a field $F$, and $b_{1}, b_{2}, b_{3} \in A$. We set

$$
a\left(b_{1}, b_{2}, b_{3}\right)=b_{3} \cdot b_{1} b_{2}+b_{2} b_{1} \cdot b_{3}-b_{2} b_{3} \cdot b_{1}-b_{1} \cdot b_{3} b_{2} .
$$

Since $A$ is alternative, we can also write

$$
a\left(b_{1}, b_{2}, b_{3}\right)=\left[b_{2},\left[b_{1}, b_{3}\right]\right]-4\left(b_{1}, b_{2}, b_{3}\right) .
$$

We define $a^{1}\left(b_{1}, b_{2}, b_{3}\right)=a\left(b_{1}, b_{2}, b_{3}\right)$ and inductively for $k>1$ and elements $b_{1}, \ldots, b_{2 k+1} \in A$

$$
a^{k}\left(b_{1}, \ldots, b_{2 k+1}\right)=a\left(a^{k-1}\left(b_{1}, \ldots, b_{2 k-1}\right), b_{2 k}, b_{2 k+1}\right) .
$$

When no ambiguity arises, we will write the left-hand side of this expression $a^{k}()$. We say that $A$ is a-nilpotent if there is a $k>0$ such that $a^{k}()=0$ for all choices $b_{i} \in A$. Clearly any subalgebra and any homomorphic image of an $a$-nilpotent alternative algebra is $a$-nilpotent. Furthermore, since $a^{k}()$ is multilinear for all $k$, $A$ is $a$-nilpotent if and only if $A_{K}$ is $a$-nilpotent for all extensions $K$ of $F$. If $A$ contains a unity and $G$ is a subfield of the centre of $A$ containing the unity of $A$, then $A$ can be regarded as an algebra over $G$. In this case, we have that $A$ is $a$-nilpotent if and only if $A / G$ is $a$-nilpotent.

(4.2) Suppose $b, c \in A$. We define a linear transformation on $A$ as follows: for all $x \in A, x S(b, c)=a(x, b, c)$. Note that $S(b, c)$ is linear in each of its arguments. If $b \in A$ and for some positive integer $n=n(b), S(b, b)^{n}=0$, then $b$ is called $a$ nilpotent. If every element of $A$ is $a$-nilpotent, $A$ is called $a$-nil. We note that if $A$ is $a$-nil and $\operatorname{dim} A=m<\infty$, then $S(b, b)^{m}=0$ for all $b \in A$.

Throughout the rest of this section and $\S 5$, we will assume that $A$ is an $m<\infty$ dimensional alternative algebra with unity 1 over a field $F$.

Our determination of the structure of $a$-nilpotent alternative algebras is parallel to the determination of the structure of associator nilpotent Jordan algebras [6]. Hence where proofs are not provided, the corresponding results in the Jordan case will be cited, the proofs in the alternative case being the same (mutatis mutandis).

(4.3) Suppose $A$ contains a nil ideal $N$ such that $A / N$ is an associative, purely inseparable algebraic field over $F$. Then $A$ is called purely inseparable. If $F$ is perfect, this condition becomes $A / N \cong F$, or $A=F \cdot 1+N$.

LEMma 4.4. If $A$ is purely inseparable and $K$ is the algebraic closure of $F$, then $A_{K}=K \cdot 1+N$ where $N$ is a nil ideal of $A_{K}$.

Proof [6, Lemma 1, p. 344].

(4.5) If $b \in A$, then $b$ is separable if the subalgebra $F[b]$ of $A$ generated by $b$ and 1 is separable, i.e. for all extensions $P$ of $F, F[b]_{P}$ is a direct sum of simple ideals. If $e$ is an idempotent of $A$, then $e$ is separable. If $b$ is separable and $K$ is the algebraic closure of $F$, then $F[b]_{K}=K \cdot e_{1}+\cdots+K \cdot e_{t}$ where $\left\{e_{1}, \ldots, e_{t}\right\}$ is a set of pairwise orthogonal idempotents whose sum is 1 . 
Lemma 4.6. Suppose $A$ is a-nilpotent. Then every separable element of $A$ is contained in the centre of $A$.

Proof [6, Lemma 2, p. 345].

THEOREM 4.7. The algebra $A$ is a-nilpotent if and only if $A=A_{1} \oplus \cdots \oplus A_{s}$ where the $A_{i}$ are ideals of $A$, and each $A_{i}$ contains a separable subfield $G_{i}$ of its centre containing the identity of $A_{i}$ such that $A_{i} / G_{i}$ is purely inseparable.

Proof [6, Theorem 18, p. 345].

Corollary 4.8. Suppose $F$ is algebraically closed. Then $A$ is a-nilpotent if and only if $A$ is expressible as a direct sum of ideals $A_{i}$ where $A_{i}=F \cdot 1_{i}+N_{i}, 1_{i}$ is the identity of $A_{i}$ and $N_{i}$ is a nil ideal of $A_{i}$.

Proof [6, Corollary 2, p. 346].

To prove the analogue of Engel's theorem, we will require

LEMMA 4.9. For any $b \in A$ and nonnegative integers $i, j, k$,

$$
S\left(b^{i}, b^{j}\right)^{k}=S(b, b)^{k} \varphi_{k}\left(R_{b}, L_{b}\right)
$$

where $\varphi_{k}(i, j)$ is a polynomial in $i$ and $j$ over the integers. Consequently, if $b$ is $a$ nilpotent, then $S\left(b^{i}, b^{j}\right)$ is a nilpotent linear transformation of $A$ for all $i, j \geqq 0$.

Proof. Since the subalgebra of $A$ generated by any two elements from $A$ is associative, it is clear that

$$
S\left(b^{i}, b^{j}\right)=S\left(b^{j}, b^{i}\right) \text { for all } i, j \geqq 0 .
$$

We recall that in an associative algebra

$$
[x y, z]=x[y, z]+[x, z] y .
$$

Applying this to (4.1), and since the subalgebra of $A$ generated by $\{x, b\}$ is associative for $x \in A$, we find $x S\left(b^{i}, b^{j}\right)=\left[b^{i-1} b,\left[x, b^{j}\right]\right]$, whence

$$
S\left(b^{i}, b^{j}\right)=S\left(b, b^{j}\right) L_{b^{i-1}}+S\left(b^{i-1}, b^{j}\right) R_{b}, \quad i \geqq 2 .
$$

From (a) and (c) an easy induction gives us

$$
S\left(b^{i}, b^{j}\right)=S(b, b) \varphi_{1}\left(R_{b}, L_{b}\right), \quad i, j \geqq 1,
$$

where $\varphi_{1}(i, j)$ is a polynomial in $i$ and $j$ over the integers. Next, using (b), we obtain

$$
R_{b} S\left(b^{i}, b^{j}\right)=S\left(b^{i}, b^{j}\right) R_{b}, \quad i, j \geqq 0,
$$

and similarly

$$
L_{b} S\left(b^{i}, b^{j}\right)=S\left(b^{i}, b^{j}\right) L_{b}, \quad i, j \geqq 0 .
$$

Now (e) and (f) together with (2.3) imply that for all $i$ and $j, S\left(b^{j}, b^{j}\right)$ commutes with any polynomial in $R_{b}$ and $L_{b}$. Thus from (d), we have

$$
S\left(b^{i}, b^{j}\right)^{k}=S(b, b)^{k} \varphi_{k}\left(R_{b}, L_{b}\right)
$$


where $i, j, k>0$ and $\varphi_{k}=\varphi_{k}(i, j)$ is a polynomial over the integers. The case when one or both of $i$ and $j$ is zero is trivial. The final assertion now follows from ( $\mathrm{g}$ ).

We now give

THEOREM 4.10 [ENGEL]. The algebra $A$ is a-nil if and only if it is a-nilpotent.

Proof [6, Theorem 19, p. 347].

5. Cartan subalgebras of alternative algebras. Suppose $L$ is a nilpotent Lie algebra of linear transformations of an $n<\infty$ dimensional vector space $W$ where the ground field of both $L$ and $W$ is $F$. Then we can write $W=W_{0} \oplus W_{1}$ where $W_{0}$ and $W_{1}$ are respectively the Fitting null and one component of $W$ relative to $L$. We have $W_{0}=\left\{w \in W \mid w h^{n}=0\right.$ for all $\left.h \in L\right\}$, and $W_{1}=\bigcap_{i} W\left(L^{*}\right)^{i}$ where $L^{*}$ is the subalgebra of the enveloping associative algebra $C(L)$ of $L$ generated by $L$ [5, p. 39]. Furthermore, Jacobson [4, p. 599] has shown that $W_{1}$ can be characterized as any complementary subspace of $W_{0}$ that is invariant under $L$.

Now suppose $P$ is an arbitrary extension of $F$. Then $L_{P}$ is a nilpotent Lie algebra of linear transformations of $W_{P}$. Since every element of $\left(L_{P}\right)^{*}$ is a $P$-linear combination of elements of $L^{*}$, and since $W_{0}\left(L^{*}\right)^{n}=0$, we have $\left(W_{P}\right)_{0}=\left(W_{0}\right)_{P}$. Similarly, we have $\left(W_{1}\right)_{P}=\left(W_{P}\right)_{1}$.

(5.1) Suppose $b \in A$. We set $B_{b}=\left\{x \in A \mid x S(b, b)^{m}=0\right\}$. Note that both $b$ and 1 are contained in $B_{b}$. $B_{b}$ is called minimal Engel in $A$ if for $c \in A$ and $B_{c} \subseteq B_{b}, B_{b}=B_{c}$.

(5.2) Suppose $R$ is a subalgebra of $A$. By $L_{A}(R)$ we mean the Lie algebra of linear transformations of $A$ generated by the set $\{S(b, c) \mid b, c \in R\}$.

We have

THEOREM 5.3. Suppose $R$ is an a-nilpotent subalgebra of $A$ and $1 \in R$. Then $L_{A}(R)$ is nilpotent, and if $A=A_{0} \oplus A_{1}$ is the Fitting decomposition of $A$ relative to $L_{A}(R)$, then

(i) $A_{0}$ is a subalgebra of $A$ containing $R$,

(ii) $A_{0} A_{1} \subseteq A_{1}$ and $A_{1} A_{0} \subseteq A_{1}$.

Moreover, if $F$ is algebraically closed and $\left\{e_{1}, \ldots, e_{t}\right\}$ is a set of pairwise orthogonal primitive idempotents of $R$ whose sum is 1 , then $A_{0}=\bigcap\left\{B_{b} \mid b \in R\right\}=\bigcap_{i=1}^{t} B_{e_{i}}$.

Proof. By the remarks preceding (5.1), we see that we may assume $F$ is algebraically closed. By virtue of Corollary 3.11 and Corollary 4.8, the proof that $L_{A}(R)$ is nilpotent is the same as Jacobson's proof of the analogous result in the Jordan case (mutatis mutandis) [4, p. 600].

We will now prove the rest of the theorem. Since $F$ is algebraically closed, we may write $R=\sum_{i=1}^{t}\left(F \cdot e_{i}+N_{i}\right)$ where $\left\{e_{1}, \ldots, e_{t}\right\}$ is a set of pairwise orthogonal primitive idempotents whose suni is 1 and the $N_{i}$ are nil ideals of $R$. Suppose $\sum_{i, j} A_{i j}$ is the Pierce decomposition of $A$ relative to the set $\left\{e_{1}, \ldots, e_{t}\right\}$. Then for all integers $i, p, q>0, A_{i i} S\left(e_{p}, e_{q}\right)=0$. It follows easily that if $a_{i i} \in A_{i i}$ and $b, c \in R$, then $a_{i i} S(b, c)=a_{i i} S\left(n_{i}, n_{i}^{\prime}\right)$ where $n_{i}, n_{i}^{\prime}$ are the components of $b$ and $c$ respectively 
in $N_{i}$. Thus $a_{i i} S(b, c) \in A_{i i}$, and hence $a_{i i} S(b, c)^{m}=a_{i i} S\left(n_{i}, n_{i}^{\prime}\right)^{m}=0$ by Corollary 3.11. Thus each element of $L_{A}(R)$ acts nilpotently on $A_{i i}$, and we have $R \subseteq \sum A_{i i} \subseteq A_{0}$. If $b \in R, S(b, b) \in L_{A}(R)$, hence $A_{0} \subseteq \bigcap\left\{B_{b} \mid b \in R\right\} \subseteq \bigcap_{i=1}^{t} B_{e_{i}}$. Now let $x \in \bigcap B_{e_{i}}$. By (2.5), we write $x=\sum_{i} x_{i i}+\sum_{i \neq j} x_{i j}$. We compute $x S\left(e_{k}, e_{k}\right)=\sum_{i=k \neq j ; i \neq j=k} x_{i j}$. But $x \in B_{e_{k}}$ means that $x S\left(e_{k}, e_{k}\right)^{m}=\sum_{i=k \neq j: i \neq j=k} x_{i j}=0$, and as (2.5) is a direct sum, $x_{i k}=x_{k i}=0$ for $i \neq k$. Since $x \in B_{e_{k}}$ for $k=1, \ldots, t$, it follows that $x=\sum x_{i i}$, i.e. $\bigcap B_{e_{i}} \subseteq \sum A_{i i}$. Consequently, $R \subseteq A_{0}=\sum A_{i i}=\bigcap\left\{B_{b} \mid b \in R\right\}=\bigcap B_{e_{i}}$. As $\sum_{i \neq j} A_{i j}$ is invariant under $L_{A}(R)$, we have $A_{1}=\sum_{i \neq j} A_{i j}$.

That $A_{0}$ is a subalgebra of $A$, and $A_{0} A_{1} \subseteq A_{1}$ and $A_{1} A_{0} \subseteq A_{1}$ follow from (2.6), and the proof is complete.

Corollary 5.4. For any $b \in A, B_{b}$ is a subalgebra of $A$.

Proof. We define $B_{b}^{*}=\left\{x \in A \mid x S\left(b^{i}, b^{j}\right)^{m}=0, i, j=0,1, \ldots\right\}$. From Lemma 4.9, we have immediately that $B_{b}=B_{b}^{*}$. We recall that $F[b]$ is the subalgebra of $A$ generated by $b$ and 1 . Clearly $F[b]$ is $a$-nilpotent. Thus $L_{A}(F[b])$ is nilpotent, and the Fitting null component $A_{0}$ of $A$ relative to $L_{A}(F[b])$ is a subalgebra of $A$ containing $F[b]$. But elements of $L_{A}(F[b])$ are sums of products of $S\left(b^{i}, b^{j}\right), i, j=0,1, \ldots$, and it follows that $B_{b}=A_{0}$, as required.

(5.5) A subalgebra $H$ of $A$ is a Cartan subalgebra if

(i) $H$ is $a$-nilpotent,

(ii) $H$ coincides with the Fitting null component of $A$ relative to $L_{A}(H)$.

We know that Cartan subalgebras exist in some alternative algebras. Indeed, if $F$ is algebraically closed, by Theorem 5.3 we have that $H$ is a Cartan subalgebra of $A$ if and only if $H$ contains a set $\left\{e_{1}, \ldots, e_{t}\right\}$ or pairwise orthogonal primitive idempotents whose sum is 1 , and $H=\sum A_{i i}$ where $\sum A_{i j}$ is the Pierce decomposition of $A$ relative to the $e_{i}$. However, to construct Cartan subalgebras in a more general situation, we will follow the work of Barnes in the Lie case [1].

Thus let $\left\{u_{1}, \ldots, u_{m}\right\}$ be a basis for $A$ over $F$, and $P=F\left\{\xi_{1}, \ldots, \xi_{m}\right\}$ be the field of rational expressions where the $\xi_{i}$ are independent transcendentals over $F$. Then $x=\sum_{i=1}^{m} \xi_{i} u_{i} \in A_{P}$ is a generic element of $A$, and the characteristic polynomial $\varphi(t, x)$ of $S(x, x)$ in $A_{P}$ can be written

$$
\varphi(t, x)=t^{m}+\beta_{1}(x) t^{m-1}+\cdots+\beta_{s}(x) t^{m-s}
$$

where $\beta_{i}(x)=\beta_{i}\left(\xi_{1}, \ldots, \xi_{m}\right)$ is a homogeneous polynomial of total degree $2 i$ or the zero polynomial, and $\beta_{s}(x)$ is the last coefficient which is not the zero polynomial. Since $x$ is an eigenvector of $S(x, x)$, we have that $s<m$. If $a=\sum_{i=1}^{m} \alpha_{i} u_{i} \in A$, then the specialization $\xi_{i}=\alpha_{i}$ in (5.6) gives

$$
\varphi(t, a)=t^{m}+\beta_{1}(a) t^{m-1}+\cdots+\beta_{s}(a) t^{m-s} .
$$

We note that $\varphi(t, a)$ is unaffected by an extension of the ground field. The element $a \in A$ is called $a$-regular if and only if $\beta_{s}(a) \neq 0$. Clearly if $a$ is an $a$-regular element of $A$, then $B_{a}$ is minimal Engel in $A$, but not necessarily vice versa. Also the property 
of being $a$-regular is preserved under field extensions. Moreover, if $F$ is infinite, the set of $a$-regular elements of $A$ forms a dense Zariski open subset of $A$.

The proofs of the following two lemmas are virtually identical with the proofs given by Barnes in the Lie case [1, p. 351].

LemmA 5.8. If $F$ has at least $2(m-1)$ elements, then $A$ contains a-regular elements.

Lemma 5.9. Suppose $F$ has at least $2 m$ elements. If $R$ is a subalgebra of $A, B_{b}$ is minimal with respect to dimension in $\left\{B_{c} \mid c \in R\right\}$, and $R \subseteq B_{b}$, then $B_{b} \subseteq B_{c}$ for all $c \in R$.

To prove the existence theorem, we will require the following lemma. First, we recall that if $K$ is an extension of $F$ and $\left\{u_{1}, \ldots, u_{m}\right\}$ is a basis for $A$ over $F$, then $\left\{1 \otimes u_{1}, \ldots, 1 \otimes u_{m}\right\}$ is a basis for $A_{K}$ over $K$. If $b \in A_{K}$, we will write $b=\sum_{i=1}^{m} \alpha_{i} u_{i}$, $\alpha_{i} \in K$.

LeMma 5.10. Suppose $F$ has at least $2 m$ elements, $R$ is a subalgebra of $A$, and for all $b \in R, S(b, b)$ is nilpotent of $A$. Then for any extension $K$ of $F, S\left(b^{\prime}, b^{\prime}\right)$ is nilpotent on $A_{K}$ for all $b^{\prime} \in A_{K}$.

Proof. Suppose $\left\{b_{1}, \ldots, b_{m^{\prime}}\right\}$ is a basis for $R$ over $F, b \in R$, and $b=\sum_{i=1}^{m^{\prime}} \beta_{i} b_{i}$ where $\beta_{i} \in F$. Since $S$ is linear in each of its arguments, we have

$$
\begin{aligned}
0 & =S(b, b)^{m} \\
& =\left(\sum_{j_{1}, j_{2}=1}^{m^{\prime}} \beta_{j_{1}} \beta_{j_{2}} S\left(b_{j_{1}}, b_{j_{2}}\right)\right)^{m} .
\end{aligned}
$$

Let $T_{i_{1}}^{\prime}$ equal the sum of the terms of $T$ where $\beta_{1}$ appears to the $i_{1}$ power, and set $T_{i_{1}}^{\prime}=\left(\beta_{1}\right)^{i_{1}} T_{i_{1}}$. Then $T=\sum_{i_{1}=0}^{2 m}\left(\beta_{i_{1}}\right)^{i_{1}} T_{i_{1}}$. However, $T_{2 m}=S\left(b_{1}, b_{1}\right)^{m}=0$ and $T_{0}=$ $S\left(\beta_{2} b_{2}+\cdots+\beta_{m^{\prime}} b_{m^{\prime}}, \beta_{2} b_{2}+\cdots+\beta_{m^{\prime}} b_{m^{\prime}}\right)^{m}=0$. Therefore,

$$
T=\sum_{i_{1}=1}^{2 m-1}\left(\beta_{1}\right)^{i_{1}} T_{i_{1}}
$$

Choosing $2 m-1$ different nonzero values for $\beta_{1}$, we obtain a system of $2 m-1$ homogeneous equations in $T_{i_{1}}, i_{1}=1, \ldots, 2 m-1$, whose matrix of coefficients has a nonzero determinant. Thus we conclude that $T_{i_{1}}=0$ for $i_{1}=0, \ldots, 2 m$.

Now let $T_{i_{1}, i_{2}}^{\prime}$ be the sum of the terms of $T_{i_{1}}$ where $\beta_{2}$ appears to the $i_{2}$ power, and set $T_{i_{1}, i_{2}}^{\prime}=\left(\beta_{2}\right)^{i_{2}} T_{i_{1}, i_{2}}$. Repeating the above process, we conclude $T_{i_{1}, i_{2}}=0$. Continuing in this fashion, we find that $T_{i_{1}, \ldots, i_{m^{\prime}}}=0$ for all $m^{\prime}$-tuples $\left(i_{1}, \ldots, i_{m^{\prime}}\right)$ such that $\sum_{j=1}^{m^{\prime}} i_{j}=2 m$.

Finally, if $b^{\prime} \in R_{K}$, we write $b^{\prime}=\sum_{i=1}^{m^{\prime}} \alpha_{i} b_{i}$ where $\alpha_{i} \in K$. Then

$$
S\left(b^{\prime}, b^{\prime}\right)^{m}=\sum_{i_{1}, \ldots, i_{m^{\prime} ; i_{1}+\cdots+i_{m^{\prime}}=2 m}} \alpha_{1}^{i_{1}} \cdots \alpha_{m^{\prime}}^{i_{m^{\prime}}} T_{i_{1}, \ldots, i_{m^{\prime}}}=0,
$$

as required. 
We have

Theorem 5.11. Suppose $F$ has at least $2 m$ elements. Then $H$ is a Cartan subalgebra of $A$ if and only if $H$ is minimal Engel in $A$.

Proof. Suppose $H=B_{b}$ is minimal Engel in $A$. Then $B_{b}$ is minimal with respect to dimension in $\left\{B_{c} \mid c \in H\right\}$, consequently, by Lemma 5.9, $B_{b} \subseteq B_{c}$ for all $c \in H$. Thus $S(c, c)$ is nilpotent on $H$ for all $c \in H$, and by Theorem 4.10, $H$ is $a$-nilpotent. Since $L_{A}(H)$ is nilpotent, $H \subseteq A_{0}$ where $A_{0}$ is the Fitting null component of $A$ relative to $L_{A}(H)$. However, $A_{0} \subseteq B_{b}=H$, and $H=A_{0}$ as desired.

To prove the converse, we first make the following observations:

(a) if $R$ is a subalgebra of $A$ and $R \subseteq B_{c}$ for some $c \in A$, then $R_{K} \subseteq\left(B_{c}\right)_{K}$ for all extensions $K$ of $F$,

(b) if $R$ is a Cartan subalgebra of $A$, then $R_{K}$ is a Cartan subalgebra of $A_{K}$ for all extensions $K$ of $F$ (see remarks preceding (5.1)).

Now suppose $H$ is a Cartan subalgebra of $A$ and $B_{b}$ is minimal with respect to dimension in $\left\{B_{h} \mid h \in H\right\}$. We claim $H=B_{b}$. Since $H$ is $a$-nilpotent, $H \subseteq B_{b}$, and consequently by Lemma 5.9, $B_{b} \subseteq B_{h}$ for all $h \in H$. If $K$ is the algebraic closure of $F$ then $H_{K}=\bigcap\left\{B_{h^{\prime}} \mid h^{\prime} \in H_{K}\right\}$. But $B_{b} \subseteq B_{h}$ for all $h \in H$ means $S(h, h)$ acts nilpotently on $B_{b}$ for all $h \in H$. By Lemma 5.10, $S\left(h^{\prime}, h^{\prime}\right)$ is nilpotent on $\left(B_{b}\right)_{K}$ for all $h^{\prime} \in H_{K}$. Therefore $\left(B_{b}\right)_{K} \subseteq H_{K}$, and by (a) it follows that $\left(B_{b}\right)_{K}=H_{K}$. Hence the dimension of $\left(B_{b}\right)_{K}$ and $H_{K}$ over $K$ are equal, which implies the dimension of $H$ and $B_{b}$ over $F$ are equal. Since $H \subseteq B_{b}, H=B_{b}$. That $B_{b}$ is minimal Engel in $A$ follows easily, as required.

COROLlary 5.12. If $a$ is an a-regular element of $A$, then $B_{a}$ is a Cartan subalgebra of $A$.

COROLlaRY 5.13. Suppose F has at least $2(m-1)$ elements. Then A contains Cartan subalgebras.

We conclude this section with some remarks on the conjugacy of Cartan subalgebras. Our discussion parallels the Jordan case, and requires many results that can be found in Chevalley [2]. We will make the assumption that the characteristic of $F$ is zero.

In this situation, $D(b, c)=\left[L_{b}, L_{c}\right]+\left[L_{b}, R_{c}\right]+\left[R_{b}, R_{c}\right]$ is a derivation of $A$ for all $b, c \in A$. The set $\{D(b, c) \mid b, c \in A\}$ of these derivations generates an ideal $D^{\prime}(A)$ in the derivation algebra $D(A)$ of $A$. Such derivations are called inner. Now let (S) $=\left\{G \mid G\right.$ is an algebraic group of linear transformations whose Lie algebra $G_{L}$ contains $\left.D^{\prime}(A)\right\}$, and set $I=\bigcap\{G \mid G \in(B)\}$. Since the group of automorphisms of $A$ is an algebraic group whose Lie algebra is $D(A)$, elements in $I$ are automorphisms of $A$. We call $I$ the inner automorphism group of $A$. We have

THEOREM 5.14. Suppose $F$ is algebraically closed and of characteristic zero. If $H_{1}$ and $H_{2}$ are two Cartan subalgebras of $A$, then there is an $\sigma \in I$ such that $H_{1}=H_{2} \sigma$. 
Proof. The proof is patterned after Chevalley's proof in the Lie case [2, III, pp. 215-219] and follows the proof in the Jordan case [4, p. 603]. We sketch it here for the reader's convenience.

We begin by showing that $H_{1}$ and $H_{2}$ each contain relatively open subsets (in the Zariski topology) $O_{1}$ and $O_{2}$ such that $T\left(\Omega_{i}: a\right)=A$ for all $a \in O_{i}$, where $\Omega_{i}$ is the orbit of $H_{i}$ under $I(A)$ and $T\left(\Omega_{i}: a\right)$ is the tangent space to $\Omega_{i}$ at $a, i=i, 2$. By the remark made after (5.5), there is a set $\left\{e_{1}, \ldots, e_{t}\right\}$ of pairwise orthogonal primitive idempotents whose sum is 1 and $H_{1}=\sum A_{i i}$ where $\sum A_{i j}$ is the Pierce decomposition relative to this set, and each $A_{i i}=F \cdot e_{i}+N_{i}$ where $N_{i}$ is a nil ideal of $H_{1}$. Let $O_{1}=\left\{a \in H_{1} \mid a=\sum\left(\alpha_{i} e_{i}+z_{i}\right), \alpha_{i} \in F, z_{i} \in N_{i}\right.$, and $\left.\prod_{i \neq j}\left(\alpha_{i}-\alpha_{j}\right) \neq 0\right\}$. Then $O_{1}$ is an open subset of $H_{1}$. If $b_{k p} \in A_{k p}, k \neq p$, and $a=\sum\left(\alpha_{i} e_{i}+z_{i}\right) \in O_{1}$, we compute

$$
a D\left(e_{k}, b_{k p}\right)=\left(\alpha_{k}-\alpha_{p}\right) b_{k p}+z_{k} b_{k p}-b_{k p} z_{p} \text {. }
$$

Since $D\left(e_{k}, b_{k p}\right) \in D^{\prime}(A), a D\left(e_{k}, b_{k p}\right) \in T\left(\Omega_{1}: a\right)$ [2, III, pp. 192-193]. Define $S_{k p}=$ $\left(\alpha_{k}-\alpha_{p}\right) M+L_{z_{k}}-R_{z_{p}}$ where $M$ is the identity transformation on $A$. Hence $b_{k p} S_{k p} \in T\left(\Omega_{1}: a\right)$. Now $S_{k p}$ maps $A_{k p}$ onto itself by (2.6). Also, by Corollary 3.11, $L_{z_{k}}-R_{z_{p}}$ is nilpotent, and as $\alpha_{k}-\alpha_{p} \neq 0, S_{k p}$ is invertible. Therefore, $A_{k p} \subseteq T\left(\Omega_{1}: a\right)$ for all $k \neq p$, and it follows that $T\left(\Omega_{1}: a\right)=A$. Similarly $T\left(\Omega_{2}: b\right)=A$ for all $b \in O_{2}$, where $O_{2}$ is defined in a manner analogous to $O_{1}$. Hence $\Omega_{1}$ and $\Omega_{2}$ contain nonempty open subsets of $A$ [2, III, Proposition 13, p. 180 and the corollary on p. 192]. By (5.7), $\Omega_{1} \cap \Omega_{2}$ contains an $a$-regular element $b$. Hence there are elements $\sigma_{i} \in I$ with $b \in H_{i} \sigma_{i}, i=1,2$. Then $H_{i} \sigma_{i} \subseteq B_{b}$, and since $B_{b}$ and $H_{i} \sigma_{i}$ are Cartan subalgebras of $A, B_{b}=H_{1} \sigma_{1}=H_{2} \sigma_{2}$. Thus $H_{1}=H_{2} \sigma$ where $\sigma=\sigma_{2} \sigma_{1}^{-1} \in I$, as desired.

6. Conclusion. Let $\mathfrak{A}$ be a class of finite-dimensional alternative, Jordan, and Lie algebras over a field $F$ where

(i) if $A \in \mathfrak{A}$ and $\Theta \in \operatorname{Hom} A$, then $A \Theta \in \mathfrak{U}$,

(ii) if $A \in \mathfrak{A}$ and $I$ is a subalgebra of $A$, then $I \in \mathfrak{A}$,

(iii) if $A \in \mathfrak{A}$, then the ground field of $A$ is either $F$ or an extension of $F$; if the ground field of $A$ is $K$, then $A_{P} \in \mathfrak{A}$ for all extensions $P$ of $K$,

(iv) if $A \in \mathfrak{A}$ and $A$ is either alternative or Jordan, then $A$ contains a unity element,

(v) if $A \in \mathfrak{A}$ and $A$ is either Jordan or Lie, then the characteristic of $F$ is different from 2.

We remark that (i)-(iii) means that $\mathfrak{A}$ is a universal class of algebras.

(6.1) Suppose $A \in \mathfrak{A}$ and $b_{1}, b_{2}, b_{3} \in A$. We set $a\left(b_{1}, b_{2}, b_{3}\right)=b_{3} \cdot b_{1} b_{2}+b_{2} b_{1} \cdot b_{3}$ $-b_{2} b_{3} \cdot b_{1}-b_{1} \cdot b_{3} b_{2}$ and $S\left(b_{2}, b_{3}\right)=R_{b_{2}} L_{b_{3}}+L_{b_{2}} R_{b_{3}}-L_{b_{2} b_{3}}-R_{b_{3} b_{2}}$. Let $S(A)=$ $\left\{S\left(b_{1}, b_{2}\right) \mid b_{1}, b_{2} \in A\right\}$. If $b \in A$ and the dimension of $A$ is $m$, then

$$
B_{b}=\left\{x \in A \mid x S(b, b)^{m}=0\right\}
$$

is minimal Engel in $A$ with respect to $S(A)$ if $B_{c} \subseteq B_{b}$ for $c \in A$ implies $B_{b}=B_{c}$.

(i) If $A \in \mathfrak{Q}$ and $A$ is alternative, then $a\left(b_{1}, b_{2}, b_{3}\right)$ gives the " $a$-theory" developed in $\S 4$. 
(ii) If $A \in \mathfrak{A}$ and $A$ is Jordan, then $a\left(b_{1}, b_{2}, b_{3}\right)=2\left(b_{1}, b_{2}, b_{3}\right)$, and it follows that $a\left(b_{1}, b_{2}, b_{3}\right)$ gives the "associator theory" as developed by Jacobson in [4].

(iii) If $A \in \mathfrak{A}$ and $A$ is a Lie algebra, then $a\left(b_{1}, b_{2}, b_{3}\right)=-2 b_{1} b_{3} \cdot b_{2}$ and $S\left(b_{1}, b_{2}\right)$ $=-2 \operatorname{ad}\left(b_{2}\right) \operatorname{ad}\left(b_{1}\right)$. Thus it is clear that $A$ is " $a$-nilpotent" if and only if $A$ is nilpotent, and $A$ is " $a$-nil" if and only if $A$ is nil. By virtue of Engel's theorem, these four concepts coincide on $A$.

(6.2) Suppose $A \in \mathfrak{A}$ and $R$ is an $a$-nilpotent subalgebra of $A$. Then $L_{S(A), A}(R)$, the Lie algebra of linear transformations on $A$ generated by $S(A)$, is nilpotent. The subalgebra $H$ of $A$ is a Cartan subalgebra relative to $S(A)$ if

(i) $H$ is $a$-nilpotent,

(ii) $H$ coincides with the Fitting null component of $A$ relative to $L_{S(A), A}(H)$.

In view of Theorem 5.11, and the results of Barnes [1] and the author [3], we have

THeOREM 6.3. Suppose $A \in \mathfrak{A}$ and the dimension of $A$ is $m$. If $F$ has at least $2 m$ elements, then $H$ is a Cartan subalgebra relative to $S(A)$ of $A$ if and only if $H$ is minimal Engel with respect to $S(A)$ in $A$.

Proof. If $A$ is either alternative or Jordan, the proof is clear from Theorem 5.11 or [3]. If $A$ is a Lie algebra, the proof follows since $H$ is a Cartan subalgebra relative to $S(A)$ of $A$ if and only if $H$ is a Cartan subalgebra in the classical sense.

(6.4) Suppose $A \in \mathfrak{A}$. If $A$ is alternative, then the definition of an $a$-regular element is given in $\S 5$, whereas if $A$ is Jordan, it is given in [3]. It is clear that if $A$ is Lie, we may define $a$-regular elements. If $A$ is Lie and $x$ is a generic element of $A$, then $x S(x, x)=0$, and we see that the analogue of Lemma 5.8 is valid for the Lie algebras in $\mathfrak{A}$. Thus we have

Corollary 6.5. If $A \in \mathfrak{A}$ and $a$ is an a-regular element of $A$, then $B_{a}$ is a Cartan subalgebra of $A$.

Corollary 6.6. Suppose $A \in \mathfrak{A}, \operatorname{dim} A=m$, and $F$ has at least $2(m-1)$ elements. Then $A$ contains Cartan subalgebras relative to $S(A)$.

(6.7) Notes. (1) It should be noted that if $A$ is an alternative algebra, then we can make $A$ into a Jordan algebra $A^{+}$by defining a new product $a \circ b$ of $A$ by the equation $a \circ b=\frac{1}{2}(a b+b a), a, b \in A$. We call $A^{+}$the symmetric algebra of $A$. Using the associator in $A^{+}$we would expect to develop a Cartan theory for $A$. However, if $b_{1}, b_{2}, b_{3} \in A, 2\left(b_{1} \circ b_{2}\right) \circ b_{3}-2 b_{1} \circ\left(b_{2} \circ b_{3}\right)=4 a\left(b_{1}, b_{2}, b_{3}\right)-4\left(b_{1}, b_{2}, b_{3}\right)$. Therefore, there is no reason to expect that the Cartan theory developed earlier for alternative algebras arises from iterating the associator in $\mathrm{A}^{+}$.

(2) The fact that in (6.2) we define Cartan subalgebras relative to a particular set " $S($ )" of linear transformations would suggest that other sets of transformations on algebras in $\mathfrak{A}$ would yield a "Cartan theory". Indeed, in the Lie case, we see that " $S($ )" and "ad" yield Cartan theories that coincide. It turns out that 
the whole concept of a "Cartan theory" can be developed for certain universal classes of algebras, the above class $\mathfrak{A}$ being an example of one such class. A more detailed study of this fact is in preparation [3].

\section{REFERENCES}

1. D. W. Barnes, On Cartan subalgebras of Lie algebras, Math. Z. 101 (1967), 350-355. MR 36 \#3837.

2. C. Chevalley, Théorie des groupes de Lie. Tomes II, III, Actualités Sci. Indust., nos. 1152, 1226, Hermann, Paris, 1951, 1955. MR 14, 448; MR 16, 901.

3. D. M. Foster, Generalizations of nilpotence and solvability in universal classes of algebras (preliminary version).

4. N. Jacobson, Cartan subalgebras of Jordan algebras, Nagoya Math. J. 27 (1966), 591-609. MR 34 \#218.

5. - Lie algebras, Interscience Tracts in Pure and Appl. Math., no. 10, Interscience, New York, 1962. MR 26 \#1345.

6. —-, Structure and representations of Jordan algebras, Amer. Math. Soc. Colloq. Publ., vol. 39, Amer. Math: Soc., Providence, R. I., 1968. MR 40 \#4330.

7. R. D. Schafer, Representations of alternative algebras, Trans. Amer. Math. Soc. 72 (1952), 1-17. MR 13, 527.

8. - An introduction to nonassociative algebras, Pure and Appl. Math., vol. 22, Academic Press, New York, 1966. MR 35 \#1643.

Michigan State University, East Lansing, Michigan 48823 